\title{
RELATIVELY INVARIANT SYSTEMS AND THE SPECTRAL MAPPING THEOREM
}

\author{
BY ROBIN HARTE
}

Communicated by Robert G. Bartle, July 27, 1972

1. Introduction. In this note we consider the extension of the spectral mapping theorem ([2], [3]) to certain noncommuting systems of elements, notably the 'quasi-commuting' systems of McCoy [5]. Full proofs and more detail are to appear elsewhere [4].

2. Relative joint spectra. Suppose $a=\left(a_{1}, a_{2}, \ldots, a_{n}\right)$ is a system of elements in a complex Banach algebra $A$, with identity 1: then the joint spectrum of $a$ with respect to $A$ is ([2]; [3, Definition 1.1]) the set $\sigma(a)$ $=\sigma_{A}^{\text {joint }}(a)$ of those systems $s=\left(s_{1}, s_{2}, \ldots, s_{n}\right)$ of complex numbers for which the system $a-s=\left(a_{1}-s_{1}, a_{2}-s_{2}, \ldots, a_{n}-s_{n}\right)$ generates a proper left, or proper right, ideal in $A$. The 'one-way' spectral mapping theorem ( $[2] ;[3$, Theorem 3.2$])$ is the inclusion

$$
f \sigma(a) \subseteq \sigma f(a),
$$

valid for an arbitrary system $a \in A^{n}$ of elements and an arbitrary system $f=\left(f_{1}, f_{2}, \ldots, f_{m}\right): A^{n} \rightarrow A^{m}$ of 'polynomials' in several variables on $A$. Equality

$$
\sigma f(a)=f \sigma(a)
$$

is attained [3, Corollary 3.3] if the system of polynomials has a 'left inverse' $g: A^{m} \rightarrow A^{n}$ for which $g(f(a))=a$, or alternatively if the system of elements is commutative ([2]; [3, Theorem 4.3]). This second case is our 'spectral mapping theorem', of which we here consider the extension.

Definition 1. The joint spectrum of $b \in A^{m}$ relative to $a \in A^{n}$ in $A$ is the set

$$
\sigma_{a=a}(b)=\{t \in \sigma(b): \exists s \in \sigma(a),(s, t) \in \sigma(a, b)\} .
$$

The idea is to offer a measurement of the failure of equality in (2.1); for example there is equality

$$
\sigma_{f(a)=f(a)}(a)=\sigma(a)
$$

AMS (MOS) subject classifications (1970). Primary 47D99, 46H99; Secondary 47A10, $47 \mathrm{~A} 60$.

Key words and phrases. Joint spectrum, spectral mapping theorem, quasicommuting system. 
for arbitrary systems of elements and of polynomials, using (2.1). Equality in (2.1) for arbitrary systems of elements and 'left invertible' polynomials is derived [3, Corollary 3.3] from the equality

$$
\sigma_{a=a} f(a)=f \sigma(a)
$$

valid without restriction. The spectral mapping theorem is derived from the result ([2]; [3, Theorem 4.2]) that if $b \in A^{m}$ is an arbitrary system of elements, and $a \in A^{n}$ a commuting system commuting with $b$, then

$$
\sigma(b)=\sigma_{a=a}(b) .
$$

To derive (2.2) for a commuting system of elements we substitute $b=f(a)$ in (2.6) and combine with (2.5).

3. Relatively invariant systems. The next idea is taken straight from the proof of (2.6):

Definition 2. The system $b \in A^{m}$ is invariant under the system $a \in A^{n}$ in $A$ if there is inclusion, for each element $a_{j}$,

and

$$
\left(\text { closure } \sum_{k=1}^{m} A b_{k}\right) a_{j} \subseteq \text { closure } \sum_{k=1}^{m} A b_{k}
$$

$$
a_{j}\left(\text { closure } \sum_{k=1}^{m} b_{k} A\right) \subseteq \text { closure } \sum_{k=1}^{m} b_{k} A
$$

if $b-t \in A^{m}$ is invariant under $a \in A^{n}$ for every system $t \in C^{m}$ of scalars then $b \in A^{m}$ is completely invariant under $a$.

For example if $t \in C^{m}$ is not in the joint spectrum $\sigma(b)$ then $b-t \in A^{m}$ is invariant under arbitrary systems $a \in A^{n}$; if $a \in A^{n}$ commutes with $b \in A^{m}$ then $b$ is completely invariant under $a$. For a fixed system $b \in A^{m}$ the set of elements $c \in A$ which leave $b$ invariant form a closed subalgebra:

Lemma 1. If $b-t \in A^{m}$ is invariant under $a \in A^{n}$ then also $b-t$ is invariant under $f(a) \in A^{p}$, for an arbitrary system of polynomials $g: A^{n+m} \rightarrow A^{p}$ there is also inclusion, for each $i$,

$$
g_{i}(a, b)-g_{i}(a, t) \in\left(\operatorname{closure} \sum_{k=1}^{m} A\left(b_{k}-t_{k}\right)\right) \cap\left(\text { closure } \sum_{k=1}^{m}\left(b_{k}-t_{k}\right) A\right) .
$$

This result is built up for sums of products of polynomials $g_{i}(a, b)=b_{k}$ and $g_{i}(a, b)=f(a)$. An immediate corollary has applications in the theory of 'operator matrices':

COROLlary 1. If $b-t \in A^{m}$ is invariant under $a \in A^{n}$ and $g: A^{n+m} \rightarrow A^{p}$ is a system of polynomials then there is logical equivalence, for $r \in C^{p}$, 


$$
(t, r) \in \sigma(b, g(a, b)) \Leftrightarrow(t, r) \in \sigma(b, g(a, t)) .
$$

Equality (2.6) holds for a commuting system $a \in A^{n}$ of elements leaving the system $b \in A^{m}$ invariant:

THEOREM 1. If $b \in A^{m}$ is completely invariant under the commuting system $a \in A^{n}$ then there is equality $\sigma(b)=\sigma_{a=a}(b)$.

The proof is the same as in the case ([2]; [3, Theorem 4.2]) in which $a \in A^{n}$ commutes with $b \in A^{m}$. Recalling the induction (on the length $n$ of the system $a \in A^{n}$ ), it is clear that it is far from necessary, here, for the system $a \in A^{n}$ to be commutative. Sufficient, for example, would be that, for each $j=1,2, \ldots, n-1$,

$$
\left(a_{1}, a_{2}, \ldots, a_{j}\right) \text { is completely invariant under } a_{j+1} .
$$

Also it is always sufficient, for equality (2.6), that there exist some system $c \in A^{p}$ satisfying

$$
\sigma(b)=\sigma_{c=c}(b) \text { and } \sigma(b, c)=\sigma_{a=a}(b, c) .
$$

We need the extension of Theorem 1 by the principle (3.6) to handle the 'quasicommuting' systems of the next section.

4. Quasicommuting systems. The idea of a 'quasicommuting' pair of matrices is due to McCoy [5]. To extend this to systems of Banach algebra elements we introduce a somewhat artificial 'commutator' for two systems of elements:

Definition 3. The commutator of $a \in A^{n}$ with respect to $b \in A^{m}$ is the system of mn elements

$$
\begin{aligned}
b a-a b=\left(b_{1} a_{1}-a_{1} b_{1}, b_{1} a_{2}-a_{2} b_{1},\right. \\
\left.\quad \ldots, b_{1} a_{n}-a_{n} b_{1}, b_{2} a_{1}-a_{1} b_{2}, \ldots, b_{m} a_{n}-a_{n} b_{m}\right) .
\end{aligned}
$$

The system $b \in A^{m}$ is said to quasicommute with the system $a \in A^{n}$ if

$$
b a-a b \in A^{m n} \text { commutes with }(a, b) \in A^{n+m} ;
$$

if this is true with $b=a$ then $a$ is called a quasicommuting system.

Observe that the relation (4.2) is symmetric in $a$ and $b$. We make frequent use of the Kleinecke-Sirokov theorem [1, Problem 184]: if $a \in A^{n}$ and $b \in A^{m}$, and if the commutator $b a-a b$ commutes either with $a \in A^{n}$ or with $b \in A^{m}$ then there is inclusion

$$
\sigma(b a-a b) \subseteq\{(0,0, \ldots, 0)\} \subseteq C^{m n} .
$$

Lemma 2. Suppose $a \in A^{n}$ and $b \in A^{m}:$ if the commutator $b a-a b$ commutes with the system a then 
$(b, b a-a b)$ is completely invariant under $a$.

If $b a-a b$ commutes with $(a, b)$ then for an arbitrary system $f: A^{m} \rightarrow A^{p}$ of polynomials

$$
(f(b), b a-a b) \text { is completely invariant under } a .
$$

The first part of this follows at once, using (4.3). For the second we associate with each polynomial $f_{i}$ a system $f_{i}^{\prime}: A^{m} \rightarrow A^{m}$ for which, whenever an element $a_{j}$ quasicommutes with the system $b \in A^{m}$,

$$
f_{i}(b) a_{j}-a_{j} f_{i}(b)=\sum_{k=1}^{m} f_{i k}^{\prime}(b)\left(b_{k} a_{j}-a_{j} b_{k}\right) .
$$

THEOREM 2. Suppose $a \in A^{n}$ and $b \in A^{m}$ : if the commutator $b a-a b$ is commutative and commutes with the system $b$ then there is equality

$$
\sigma(b)=\sigma_{b a-a b=b a-a b}(b) .
$$

If instead the system $a$ is quasicommutative and commutes with ba-ab then there is equality

$$
\sigma(b, b a-a b)=\sigma_{a=a}(b, b a-a b) .
$$

If $a \in A^{n}$ is quasicommutative and quasicommutes with $b \in A^{m}$, and if $\mathrm{g}: A^{n+m} \rightarrow A^{p}$ is a system of polynomials, then there is equality

$$
\sigma g(a, b)=\sigma_{a=a} g(a, b) .
$$

The first part (4.7) follows straight from Theorem 1. For a single element $a=a_{1}$ the second part (4.8) uses Theorem 1, together with the easy part (4.4) of Lemma 2 ; then we proceed by induction on $n$, using the argument of (3.6).

Towards (4.9) it is another application of Theorem 1 that there is equality $\sigma g(a, b)=\sigma_{b a-a b=b a-a b} g(a, b)$, since our assumptions make $b a-a b$ commutative and commute with $g(a, b)$. Also for a single element $a=a_{1}$ we apply the second part (4.5) of Lemma 2, with $(b, a)$ in place of $b$, to see that $(g(a, b), b a-a b)$ is completely invariant under $a$, and again apply Theorem 1, to obtain $\sigma(g(a, b), b a-a b)=\sigma_{a=a}(g(a, b)$, $b a-a b)$. Another induction on $n$, and then (3.6), establish (4.9).

COROLlaRY 2. If $a \in A^{n}$ is quasicommutative and $f: A^{n} \rightarrow A^{m}$ is a system of polynomials then there is equality $\sigma f(a)=f \sigma(a)$.

For substitute $g(a, b)=f(a)$ in (4.9) and use (2.5).

The extension of (2.6) to quasicommutative systems is clear from (4.7) and (4.8), and uses only the first part (4.4) of Lemma 2. We are unable to simply substitute $b=f(a)$ at this stage because the system $f(a)$ does not always quasicommute with a quasicommuting system $a \in A^{n}$. 
If $A$ is the algebra of 'upper triangular' $q \times q$ matrices then it is apparent [3, Example 2.3] that the conclusions of Theorem 2 and Corollary 2 hold for arbitrary systems $a \in A^{n}$ and $b \in A^{m}$ : it follows that neither the conditions for (4.7) nor the conditions for (4.8) are necessary for (4.9). Equally neither condition is separately sufficient:

EXAMPLE. In the algebra of all $3 \times 3$ complex matrices take

$$
U=\left(\begin{array}{lll}
0 & 1 & 0 \\
0 & 0 & 1 \\
0 & 0 & 0
\end{array}\right), \quad V=\left(\begin{array}{rrr}
1 & 0 & 0 \\
0 & 0 & 0 \\
0 & 0 & -1
\end{array}\right) \text { and } W=\left(\begin{array}{rrr}
1 & 1 & 0 \\
0 & 0 & -1 \\
0 & 0 & -1
\end{array}\right) \text { : }
$$

then $V U-U V=U$ but $\sigma(V) \neq \sigma_{U=U}(V)$. Also $W=V U+U V+V$ but $\sigma(W) \neq \sigma_{V=V}(W)$.

We are unable to settle whether or not the conditions for (4.7) are sufficient for equality $(2.6)$; recall for example $[6, \S 2-3]$ the derivation of (2.6) when $a=a_{1}$ and $b a-a b=b$.

\section{REFERENCES}

1. P. R. Halmos, A Hilbert space problem book, Van Nostrand, Princeton, N.J., 1967. MR 34 \# 8178.

2. R. E. Harte, The spectral mapping theorem in several variables, Bull. Amer. Math. Soc. 78 (1972), 870-874.

3. - Spectral mapping theorems, Proc. Roy. Irish Acad. 72 (1972), 89-107.

4. - The spectral mapping theorem for quasicommuting systems, Proc. Roy. Irish Acad. (to appear).

5. N. H. McCoy, On quasi-commutative matrices, Bull. Amer. Math. Soc. 36 (1934), 327-340.

6. W. Miller, Lie theory and special functions, Math. in Sci. and Engineering, vol. 43, Academic Press, New York, 1968. MR 41 \# 8736.

Department of Mathematics, University College, Cork, Ireland 